\title{
Alcune ricerche di geometria non euclidea.
}

\author{
(Di Luigr Branchi, a Pisa.)
}

$\grave{\mathrm{E}}$

noto come le ultime ricerche di WeIvasten sul problema fondamentale dell'applicabilità riducano la determinazione di tutte le superficie applicabili sopra una superficie data a quella delle superficie integrali di una equazione a derivate parziali del secondo ordine della forma d'AMpère:

$$
\frac{\partial^{2} q}{\partial p^{2}}+\frac{\partial^{2} q}{\partial p \partial q}\left(r_{1}+r_{2}\right)+\frac{\partial^{2} \varphi}{\partial q^{2}} \cdot r_{1} r_{2}=0
$$

In questa formola $\dot{r}_{1}, r_{2}$ indicano i raggi principali di curvatura della superficie e i parametri $p, q$ denotano rispettivamente la distanza dell'origine dal piano tangente e il semi-quadrato della distanza dell'origine stessa dal punto di contatto, mentre $\varphi(p, q)$ è una funzione fissa assegnata di $p, q\left(^{*}\right)$.

Nella presente Memoria, cercando di estendere il metodo di Weingarten alla geometria degli spazi di curvatura costante, considero le superficie integrali di un'equazione della forma $(A)$, avendo attualmente $r_{1}, r_{2}$ il significato di raggi ridotti di curvatura e $p, q$ denotando rispettivamente il seno e il coseno (circolari od iperbolici secondo che la curvatura dello spazio è positiva o negativa) delle distanze di un punto fisso dal piano tangente della superficie e dal suo punto di contatto. Dalle superficie integrali di una tale

(2) Vedi lo Homorio di Vexicirtwa:

1." Su' la théorie des surfaces applicables sur une surface domée (Comptes Rendus de l'Académic de Paris, tom. CXII, pag. 607 et 706$)$.

2." Sur la léformation des surfaces (Múmoiro couronné). Acta Math, tom. 20, pagina 159. Un'esposizione del nuovo metodo di Wervacrten è data da Darboux nel tom. IV delle Lecons, ecc., pag. 308 s. s. 
equazione si deduce, con quadrature, una classe completa di superficie applicabili nello spazio ordinario (euclideo).

Diciamo subito che per tal modo il metodo di WeIngarten non viene affatto cangiato quanto all'effettivo contenuto, poichè se delle superficie dello spazio curvo, integrali della equazione fondamentale, si considerano le superficie immagini nelle consuete rappresentazioni conformi dello spazio ellittico ed iperbolico sullo spazio euclideo, queste soddisfano alla loro volta ad una medesima equazione $(A)$ di Weingarten e la classe di superficie applicabili dedotte coincide con quella fornita dall'applicazione del metodo di Weingarten nella sua forma ordinaria (vedi $\S 4$ della Memoria). Però questa trasformazione di formole si presta, come si vedrà, a varie interessanti applicazioni; fra queste basti qui citare la principale, che forma l'oggetto della presente Memoria e ciò̀ : la determinazione in termini finiti delle superficie dello spazio (iperbolico) a curvatura $K=-\frac{1}{R^{2}}$, i cui raggi principali r $i$ dotti di curvatura $r_{3}, r_{2}$ soddisfano l'equazione:

$$
\frac{1}{r_{1}}+\frac{1}{r_{2}}= \pm \frac{2}{R}
$$

e di quelle che soddisfano l'altra:

$$
r_{1}+r_{2}= \pm 2 R
$$

Esse si ottengono, con sole operazioni di derivazione, dalle superficie dello spazio euclideo d'elemento lineare:

$$
d s^{2}=d u^{2}+\left(2 u+2 v \pm e^{2 v}\right) d v^{2}
$$

superficie che il nuovo metodo di WeIngarten ha fatto conoscere completamente.

Le superficie $(B)$ dello spazio iperbolico, che vengono così ad essere tutte note, godono di una proprietà caratteristica, che le ravvicina alle ordinarie superficie minime. Se conduciamo per un punto fisso i due raggi paralleli, nel senso non euclideo, alle singole normali della superficie ed intercettiamo questi raggi con una sfera avente il centro nel punto fisso, otteniamo due rappresentazioni sferiche della superficie, che costituiscono, per lo spazio iperbolico, la naturale estensione della rappresentazione sferica di Gauss. Ora, se la superficie appartiene alla classe $(B)$, ha luogo la proprieta caratteristica che una delle due rappresentazioni sferiche riesce conforme. Sulle superficie $(B)$ le linee di curvatura costituiscono inoltre un sistema isotermo e la stessa pro- 
prietà compete evidentemente, nella rappresentazione conforme, alle loro immagini sferiche.

Possiamo trasportare questi risultati dalle superficie $(B)$ dello spazio iperbolico alle loro immagini nello spazio enclideo, p. es. in quella rappresentazione conforme dello spazio iperbolico sull'euclideo, nella quale l'elemento lineare dello spazio curvo è dato da:

$$
d s^{2}=R^{2} \frac{d x^{2}+d y^{2}+d z^{2}}{z^{2}} .
$$

Allora otteniamo in termini finiti le superficie che soddisfano alla equazione del secondo ordine:

$$
\frac{\left(1+q^{2}\right) r-2 p q s+\left(1+p^{2}\right) t}{\left(1+p^{2}+q^{2}\right)^{\frac{3}{2}}}+\frac{2}{z}\left\{\frac{1}{\sqrt{1+p^{2}+q^{2}}} \pm 1\right\}=0,
$$

la quale risulta così completamente integrata. Tali superficie costituiscono una nuova classe di superficie a linee di curvatura isoterme. Questo trovasi gì dimostrato in un mio antico lavoro $\left(^{*}\right)$; ma ciò che qui aggiungiamo di nuovo è la determinazione effettiva di tutte queste superficie, risultato non privo di d'interesse, ove si consideri la diffcoltà del problema che ha per oggetto la ricerca generale delle superficie a linee di curvatura isoterme.(**). In fine noterò come la proprietà citata di rappresentazione sferica, che godono nello spazio iperbolico le superficie $(B)$, si traduee per le loro immagini $(D)$ nella seguente proprietà caratteristica. Per ogni punto $M$ di una superficie $S$ integrale della $(D)$ conduciamo il circolo normale ad $S$ ed al piano $z=0$, e siano $A, A^{\prime}$ i punti d'incontro di detto circolo col piano. Se al punto $M$ di $S$ riguardiamo come corrispondente sul piano $z=0$ il punto $A$, ovvero $A^{\prime}$, otteniamo una doppia rappresentazione della superficie sul piano. Una delle due rappresentazioni di $S$ sul piano conserva gli angoli. Tale proprietà appartiene esclusivamente alle superficie $S$ integrali della $(D)$ ed alle sfere.

(*) Sulle superficie d'area minima negli spasi di curvatura costante (Atti dei Lincai, 1888).

(**) Cfr. Darboux, Leegons, tom. II, pag. 230 e tom. IV, pag. 217. 


\section{Formole fondamentali per la teoria delle superficie in geometria ellittica ed iperbolica.}

Comineiamo dal richiamare le formole fondamentali della teoria delle superficie nello spazio ellittico ed jperbolico $\left(^{*}\right)$. Se si tratta dello spazio ellittico, a curvatura costante positiva $K=+\frac{1}{K^{2}}$, stabiliamo in questo spazio un sistema normale di coordinate (coordinate di Weierstrass):

$$
x_{0}, x_{1}, x_{2}, x_{3}
$$

legate fra loro dall'identità quadratica:

$$
x_{0}^{2}+x_{1}^{2}+x_{2}^{2}+x_{3}^{2}=1,
$$

e l'elemento lineare dello spazio sarà dato da:

$$
d s^{2}=R^{2}\left(d x_{0}^{2}+d x_{1}^{2}+d x_{2}^{2}+d x_{3}^{2}\right) .
$$

Consideriamo in questo spazio una superficie $S$, definita assumendo le coordinate $x_{i}$ di un suo punto mobile funzioni di due parametri o coordinate curvilinee $u, v$ e introduciamo i coseni di direzione della normale ad $S$ nel punto $\left(x_{i}\right)$, o meglio le coordinate:

$$
\xi_{0}, \xi_{1}, \xi_{2}, \xi_{3},
$$

del piano tangente definite (a meno del segno) dalle relazioni:

$$
\left.\begin{array}{ll}
\frac{\mathbf{y}}{i} \xi_{i}^{2}=1, & \sum_{i} \xi_{i} x_{i}=0, \\
\sum_{i} \xi_{i} \frac{\partial x_{i}}{\partial u}=0, & \sum_{i} \xi_{i} \frac{\partial x_{i}}{\partial v}=0 .
\end{array}\right\}
$$

Alla superficie $S$ appartengono le due forme quadratiche differenziali fon-

(*) Quoste formole furono da me sviluppate in meorso di lezioni dellamo 1804-05 all'Università di Pisa o consegnate in un manoscritto per servire d'appendice all'edizione tedesca delle mie Lezioni di geometria differenziale; ma la soconda parto di queste Lezioni, per ragioni dipendenti dall'cditore, non a ancora pubblicata. 
damentali :

$$
\begin{aligned}
& d s^{2}=R^{2} \sum_{i} d x_{i}^{2}=E d u^{2}+2 F d u d v+G d v^{2} \\
& -\sum_{i} d x_{i} d \xi_{i}=D d u^{2}+2 D^{\prime} d u d v+D^{\prime \prime} d v^{2},
\end{aligned}
$$

dalle quali, come nell'ordinaria geometria euclidea, unicamente dipende la forma della superficie. I loro sei coefficienti :

$$
\begin{array}{lll}
E, & F, & G, \\
D, & D^{\prime}, & D^{\prime \prime},
\end{array}
$$

sono legati da tre relazioni, due delle quali sono le equazioni stesse di CoDAzzı come hanno luogo nello spazio euclideo $\left(^{*}\right)$ e la terza è l'equazione di Gauss modificata, a causa della curvatura dello spazio, colla formola :

$$
\frac{D D^{\prime \prime}-D^{\prime 2}}{E G-F^{2}}=k-K=k-\frac{1}{R^{2}},
$$

denotando qui $k$ la curvatura della prima forma fondamentale o, come diciamo, la curvatura assoluta della superficie. L'espressione:

$$
\frac{D D^{\prime \prime}-D^{\prime 2}}{E G-F^{2}}
$$

che eguaglia il prodotto $\frac{1}{r_{1} r_{2}}$ delle curvature principali ridotte si dirà invece la curvatura relativa; per la (5) essa è legata alla curvatura assoluta $k$ dalla formola :

$$
\frac{1}{r_{1} r_{2}}=k-\frac{1}{R^{2}} \text {. }
$$

Viceversa, se due forme quadratiche fondamentali (4) soddisfano le equazioni di Codazzi e la (5), esiste nello spazio ellittico una corrispondente superficie $S$, pienamente determinata a meno di movimenti nello spazio. Per determinare effettivamente la superficie corrispondente a due date forme quadratiche fondamentali, abbiamo da integrare il seguente sistema completamente

(ii) Vedi Lesioni, pag. 91 s. 3. 
integrabile di equazioni differenziali :

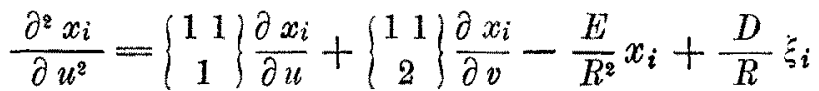

$$
\begin{aligned}
& \frac{\partial^{z} x_{i}}{\partial u^{\prime} \partial v}=\left\{\begin{array}{c}
12 \\
1
\end{array}\right\} \frac{\partial x_{i}}{\partial u}+\left\{\begin{array}{c}
12 \\
2
\end{array}\right\} \frac{\partial x_{i}}{\partial v}-\frac{F}{R^{2}} x_{i}+\frac{D^{\prime}}{R} \xi_{i} \\
& \frac{\partial^{2} x_{i}}{\partial v^{2}}=\left\{\begin{array}{c}
22 \\
1
\end{array}\right\} \frac{\partial x_{i}}{\partial u}+\left\{\begin{array}{c}
22 \\
2
\end{array}\right\} \frac{\partial x_{i}}{\partial v}-\frac{G}{R^{2}} x_{i}+\frac{D^{\prime \prime}}{R} \xi_{i} \\
& (i=0,1,2,3) \\
& \frac{\partial \xi_{i}}{\partial u}=R\left[\frac{F D^{\prime}-G D}{E G-F^{2}} \frac{\partial x_{i}}{\partial u}+\frac{F D-E D^{\prime}}{E G-F^{2}} \frac{\partial x_{i}}{\partial v}\right] \\
& \left.\frac{\partial \xi_{i}}{\partial v}=R\left[\frac{F D^{\prime \prime}-G D^{\prime}}{E G-F^{2}} \frac{\partial x_{i}}{\partial u}+\frac{F^{\prime} D^{\prime}-E D^{\prime \prime}}{E G-F^{2}} \frac{\partial x_{i}}{\partial v}\right], \quad\right\}
\end{aligned}
$$

dove $\mathrm{i}$ simboli $\left\{\begin{array}{c}i k \\ l\end{array}\right\}$ di Chrjstoffer s'intendono costruiti per la prima forma fondamentale.

Per lo spazio iperbolico, a curvatura costante $K=-\frac{1}{R^{2}}$, valgono le formole analoghe seguenti. In primo luogo le coordinate:

$$
x_{0}, x_{1}, x_{2}, x_{3}
$$

di punto sono qui legate dall'identità :

$$
x_{0}^{2}-x_{1}^{2}-x_{2}^{2}-x_{3}^{2}=1,
$$

e l'elemento lineare dello spazio è dato da:

$$
d s^{2}=R^{2}\left(d x_{1}^{2}+d x_{2}^{2}+d x_{3}^{2}-d x_{0}^{2}\right) .
$$

Per una superficie $S$ le coordinate:

$$
\xi_{0}, \xi_{1}, \xi_{2}, \xi_{3},
$$

del piano tangente sono definite dalle relazioni:

$$
\begin{array}{ll}
\sum_{i}^{1} \xi_{i}^{3}-\xi_{0}^{2}=1, & \sum_{i}^{1} \xi_{i}^{3} x_{i}-\xi_{0} x_{0}=0, \\
\sum_{i}^{1} \xi_{i} \frac{\partial x_{i}}{\partial u}-\xi_{0} \frac{\partial x_{0}}{\partial u}=0, & \sum_{i}^{1 \ldots^{3}} \xi_{i} \frac{\partial x_{i}}{\partial v}-\xi_{0} \frac{\partial x_{0}}{\partial v}=0,
\end{array}
$$


e le due forme quadratiche fondamentali dalle altre:

$$
\left.\begin{array}{l}
d s^{2}=R^{2}\left[\sum_{i}^{1} d x_{i}^{3}-d x_{0}^{2}\right]=E d u^{2}+2 F d u d v+G d v^{2} \\
d x_{0} d \xi_{0}-\sum_{i}^{1} d x_{i} d \xi_{i}=D d u^{2}+2 D^{\prime} d u d v+D^{\prime \prime} d v^{2} .
\end{array}\right\}
$$

Le relazioni che legano i sei coefficienti :

$$
E, F, G, D, D^{\prime}, D^{\prime \prime},
$$

sono ancora le equazioni di Codazzi e l'equazione di Gauss, la quale nel caso attuale si scrive:

$$
\frac{D D^{\prime \prime}-D^{\prime 2}}{E G-F^{2}}=k+\frac{1}{R^{2}} .
$$

Date le due forme quadratiche fondamentali di una superficie $S$ nello spazio jperbolico, per trovare in termini finiti la $S$ dobbiamo integrare un sistema di equazioni differenziali perfettamente analogo al sistema (6), (7) e che si deduce da questo semplicemente cangiando, nei secondi membri delle (6), i segni del termine lineare in $x_{i}$.

Osserviamo il caso speciale in cui le linee $u, v$ della superficie $S$ siano, nello spazio ellittico od iperbolico, le linee di curvatura. Allora sussistono le formole di RoDriguez:

$$
(i=0,1,2,3), \quad \frac{\partial x_{i}}{\partial u}=r_{2} \frac{\partial \xi_{i}}{\partial u}, \quad \frac{\partial x_{i}}{\partial v}=r_{1} \frac{\partial \xi_{i}}{\partial v},
$$

ove $r_{1}, r_{2}$ indicano i raggi principali ridotti di curvatura ed avendosi:

$$
D=-\frac{E}{r_{2}}, \quad D^{\prime}=0, \quad D^{\prime \prime}=-\frac{G}{r_{1}},
$$

le formole di Codazzi diventano le ordinarie formole di geometria euclidea $\left({ }^{*}\right)$ :

$$
\left.\begin{array}{l}
\left(\frac{1}{r_{1}}-\frac{1}{r_{2}}\right) \frac{\partial \log \sqrt{E}}{\partial v}-\frac{\partial}{\partial v}\left(\frac{1}{r_{2}}\right)=0 \\
\left(\frac{1}{r_{1}}-\frac{1}{r_{2}}\right) \frac{\partial \log \sqrt{G}}{\partial u}+\frac{\partial}{\partial u}\left(\frac{1}{r_{1}}\right)=0,
\end{array}\right\}
$$

(*) Cf1. Leisioni, pag. $\because 25$. 
mentre l'equazione di Gauss si scrive:

$$
\frac{1}{r_{1} r^{2}}=k \mp \frac{1}{R^{2}}
$$

valendo il segno superiore per lo spazio ellittico, l'inferiore per l'iperbolico.

\section{$\S 2$}

\section{Passaggio dalle coordinate di Weierstrass a quelle di Riemann.}

Nelle ricerche seguenti dovremo ancora servirci di quella rappresentazione conforme dello spazio ellittico ed iperbolico sull'euclideo, che si lega alla forma tipica data da Riemann per l'elemento lineare degli spazi a curvatura costante. Scriveremo questa forma tipica cosi :

$$
d s^{2}=R^{*} \frac{d x^{2}+d y^{2}+d z^{2}}{\left(x^{2}+y^{2}+z^{2} \pm \frac{1}{4}\right)^{2}},
$$

il doppio segno distinguendo il caso ellittico dall'iperbolico. Diremo $x, y, z$ coordinate di Rremans ed importerà anzi tutto che scriviamo le formole di passaggio dalle coordinate di Riemann a quelle di Weierstrass. Per lo spazio ellittico tali formole si scrivono:

$$
\left.\begin{array}{c}
x_{0}=\frac{\frac{1}{4}-\left(x^{2}+y^{2}+z^{2}\right)}{\frac{1}{4}+\left(x^{2}+y^{2}+z^{2}\right)}, \quad x_{1}=\frac{x}{\frac{1}{4}+x^{2}+y^{2}+z^{2}}, \\
x_{2}=\frac{y}{\frac{1}{4}+x^{2}+y^{2}+z^{2}}, \quad x_{3}=\frac{z}{\frac{1}{4}+x^{2}+y^{2}+z^{2}},
\end{array}\right\}
$$

e per lo spazio iperbolico avremo invece:

$$
\begin{aligned}
& x_{0}=\frac{x^{2}+y^{2}+z^{2}+\frac{1}{4}}{x^{2}+y^{2}+z^{2}-\frac{1}{4}}, \quad x_{1}=\frac{x}{x^{2}+y^{2}+z^{2}-\frac{1}{4}}, \\
& x_{2}=\frac{y}{x^{2}+y^{2}+z^{2}-\frac{1}{4}}, \quad x_{3}=\frac{z}{x^{2}+y^{2}+z^{2}-\frac{1}{4}}
\end{aligned}
$$


Consideriamo ora nello spazio ellittico od jperbolico una superficie $S$ per la quale, riferendola a coordinate di Weierstrass, manteniamo le notazioni del $\S 1$. Se la riferiamo a coordinate di Rremanx, e la riguardiamo quindi come esistente nello spazio euclideo rappresentativo, indicheremo nel modo consueto con :

$$
X, Y, Z,
$$

i coseni di direzione della sua normale e, adottando le notazioni di WeINGARTEN, porremo:

$$
p=x X+y Y+z Z, \quad 2 q=x^{2}+y^{2}+z^{2}
$$

sicchè $p$ rappresenta la distanza dell'origine dal piano tangente di $S$ e $2 q$ il quadrato della distanza dal punto di contatto. Possiamo allora dare alle formole (10), $\left(10^{*}\right)$ e a quelle che definiscono in coordinate di Weierstrass la posizione del piano tangente la forma seguente:

$$
\left.\begin{array}{l}
x_{0}=\frac{\frac{1}{4}-2 q}{\frac{1}{4}+2 q}, x_{1}=\frac{x}{\frac{1}{4}+2 q}, \quad x_{2}=\frac{y}{\frac{1}{4}+2 q}, \quad x_{3}=\frac{z}{\frac{1}{4}+2 q}, \\
\xi_{n}=\frac{p}{\frac{1}{4}+2 q}, \quad \xi_{1}=\frac{2 p x}{\frac{1}{4}+2 q}-X, \xi_{2}=\frac{2 p y}{\frac{1}{4}+2 q}-Y, \xi_{3}=\frac{2 p z}{\frac{1}{4}+2 q}-Z
\end{array}\right\}
$$

che vale per lo spazio ellittico e l'altra:

$$
\left.\begin{array}{l}
x_{0}=\frac{2 q+\frac{1}{4}}{2 q-\frac{1}{4}}, x_{1}=\frac{x}{2 q-\frac{1}{4}}, \quad x_{2}=\frac{y}{2 q-\frac{1}{4}}, \quad x_{3}=\frac{z}{2 q-\frac{1}{4}}, \\
\check{\xi}_{0}=\frac{p}{2 q-\frac{1}{4}}, \quad \xi_{1}=\frac{2 p x}{2 q-\frac{1}{4}}-X, \check{\zeta}_{2}=\frac{2 p y}{2 q-\frac{1}{4}}-Y, \check{亏}_{3}=\frac{2 p z}{2 q-\frac{1}{4}}-Z
\end{array}\right\}
$$

che vale invece per lo spazio iperbolico. 


\section{$\S 3$.}

11 metodo di Weingarten in geometria ellittica ed iperbolica.

Nelle formole fondamentali della geometria ellittica ed iperbolica, dove faremo d'ora innanzi per semplicità $R=1$, prendiamo per parametri $u, v$ rispettivamente $x_{0}$, $\xi_{0}$ e poniamo:

$$
x_{0}=\alpha, \quad \xi_{0}=\beta,
$$

e le (7) che scriviamo:

$$
\begin{gathered}
\frac{\partial \xi_{i}}{\partial \alpha}=M \frac{\partial x_{i}}{\partial \alpha}+N \frac{\partial x_{i}}{\partial \beta} \\
(i=0,1,2,3) \\
\frac{\partial \xi_{i}}{\partial \beta}=P \frac{\partial x_{i}}{\partial x}+Q \frac{\partial x_{i}}{\partial \beta}
\end{gathered}
$$

dovendo essere soddisfatte per $i=0$ dànno intanto:

indi :

$$
M=0, \quad P=1,
$$

$$
D=-N F, \quad D^{\prime}:=-N G=-(E+Q F), \quad D^{\prime \prime}=-\left(F^{\prime}+Q G\right) .
$$

$\mathrm{Ma}$, indicando con $r_{s}, r_{\mathrm{a}}$ i raggi principali ridotti di curvatura, abbiamo:

$$
\begin{gathered}
\frac{1}{r_{1} r_{2}}=\frac{D D^{\prime \prime}-D^{\prime 2}}{E G-H^{\prime 2}}=-N \\
\frac{1}{r_{1}}+\frac{1}{r_{2}}=\frac{2 F D^{\prime}-E D^{\prime \prime}-G D}{E G-F^{2}}=Q,
\end{gathered}
$$

e perd, nelle attuali coordinate $\alpha, \beta$, le formole fondamentali (7) diventano:

$$
\left.\begin{array}{l}
\frac{\partial \dot{\xi}_{i}}{\partial \alpha_{0}}=-\frac{1}{r_{1} r_{2}} \frac{\partial x_{i}}{\partial \beta} \\
\frac{\partial \dot{\xi}_{i}}{\partial \beta}=\frac{\partial x_{i}}{\partial \alpha}+\left(\frac{1}{r_{1}}+\frac{1}{r_{2}}\right) \frac{\partial x_{i}}{\partial \beta} .
\end{array}\right\}
$$

Supponiamo ora che, essendo $\varphi(\alpha, \beta)$ un'assegnata funzione di $\alpha, \beta$, i raggi 
principali di curvatura $r_{1}, r_{2}$ della $S$ soddisfino l'equazione:

$$
\frac{\partial^{2} \varphi}{\partial \alpha^{2}}+\frac{\partial^{2} \varphi}{\partial \alpha \partial \beta}\left(\frac{1}{r_{1}}+\frac{1}{r_{2}}\right)+\frac{\partial^{2} \varphi}{\partial \psi^{2}} \frac{1}{r_{1} r_{2}}=0
$$

allora le tre espressioni :

$$
\begin{aligned}
& d y_{1}=x_{1} d\left(\frac{\partial \varphi}{\partial \alpha}\right)+\xi_{1} d\left(\begin{array}{l}
\partial \varphi \\
\partial \dot{\beta}
\end{array}\right) \\
& d y_{2}=x_{2} d\left(\begin{array}{l}
\partial \phi \\
\partial \alpha \\
\partial \alpha
\end{array}\right)+\xi_{2} d\left(\frac{\partial p}{\partial \beta}\right) \\
& d y_{3}=x_{3} d\left(\frac{\partial \varphi}{\partial \alpha}\right)+\xi_{3} d\left(\frac{\partial \varphi}{\partial \beta}\right),
\end{aligned}
$$

saranno, in virtù delle (12), (13), differenziali esatti. Interpretiamo $y_{1}, y_{2}, y_{3}$, calcolate dalle (14), come coordinate cartesiane ortogonali di un punto dello spazio euclideo ed avremo un'ordinaria superficie $\Sigma$, della quale vogliamo ora calcolare l'elemento lineare. Distinguendo percio il caso dello spazio ellittico od iperbolico, otterremo nel primo caso, a causa delle (1), (3) § 1 , per l'elemento lineare della $\Sigma$ la formola:

$d s^{2}=\sum_{i} d y_{i}^{2}=\left(1-\alpha^{2}\right)\left(d \cdot \frac{\partial \varphi}{\partial \alpha}\right)^{2}-2 \alpha \beta d \cdot \frac{\partial \varphi}{\partial \alpha} d \cdot \frac{\partial \varphi}{\partial \beta}+\left(1-\beta^{2}\right)\left(d \frac{\partial \varphi}{\partial \beta}\right)^{2}$.

Nel caso iperbolico invece le $\left(1^{*}\right),\left(3^{*}\right) \S 1$ ci dànno $\left(^{*}\right)$ :

$$
d s^{2}=\left(\alpha^{2}-1\right)\left(d \cdot \frac{\partial \varphi}{\partial \alpha}\right)^{2}+2 \alpha \beta d \cdot \frac{\partial \varphi}{\partial z} d \cdot \frac{\partial \varphi}{\partial \beta}+\left(1+\beta^{2}\right)\left(d \cdot \frac{\partial \varphi}{\partial \beta}\right)^{2} .
$$

(*) Osservizione. Nel caso iperbolico potremmo applicare il metodo di WaINGarTeN anche ponendo p. e.:

$$
x_{1}=\alpha, \quad \xi_{1}=\beta,
$$

$e$ in tal caso avremmo i tre differenziali esatti:

da cui :

$$
\begin{aligned}
& d y_{0}=x_{0} d \cdot \frac{\partial \varphi}{\partial \alpha}+\xi_{0} d \cdot \frac{\partial \varphi}{\partial \beta} \\
& d y_{2}=x_{2} d \cdot \frac{\partial \varphi}{\partial \alpha}+\xi_{2} d \cdot \frac{\partial \varphi}{\partial \beta} \\
& d y_{3}=x_{3} d \cdot \frac{\partial \varphi}{\partial \alpha}+\xi_{3} d \cdot \frac{\partial \varphi}{\partial \beta}
\end{aligned}
$$

$d y_{2}{ }^{2}+d y_{3}{ }^{2}-d y_{0}^{2}=-\left(1+\alpha^{2}\right)\left(d \cdot \frac{\partial \varphi}{\partial x}\right)^{2}-2 \alpha \cdot \beta d \cdot \frac{\partial p}{\partial z} d \cdot \frac{\partial \varphi}{\partial \beta}+\left(1-\beta^{2}\right)\left(d \cdot \frac{\partial \varphi}{\partial \alpha}\right)^{2}$.

$\mathrm{Si}$ otterrebbe cosi una classe completa di superficie applicabili nello spazio parabolica indefinito di elemento lineare:

$$
d s^{2}=d x^{2}+d y^{2}-d z^{2},
$$


In ambedue $\mathrm{i}$ casi l'elemento lineare della $\Sigma$ dipende unicamente, come si vede, dalla funzione $c(\alpha, \beta)$. Ne risulta che tutte le superficie $S$ dello spazio ellittico od iperbolico, integrali della (13), dànno per quadrature, mediante le (14), superficie $\Sigma$ dello spazio ordinario applicabili l'una sull'altra. Inversamente è facile vedere che ogni superficie $\Sigma$ dello spazio euclideo di elemento lineare (15) o $\left(15^{*}\right)$ deducesi, nel modo indicato, da una superficie $S$, integrale della (31), dello spazio ellittico od iperbolico.

\section{$\S 4$.}

Paragone col metodo di Weingartin sotto la forma ordinaria.

Se trasformiamo i risultali del paragrafo precedente dalle coordinate di Weierstrass a quelle di Riemans, secondo le formole del $\$ 2$, e riguardiamo in luogo delle superficie $S$ dello spazio curvo integrali della (13) le loro immagini $S^{\prime}$ nello spazio euclideo, facilmente dimostriamo che il metodo esposto coincide sostanzialmente coll' ordinario metodo di Weingarten. Considerando ad esempio il caso ellittico, sostituiamo nelle (14) ad $x_{1}, x_{2}, x_{3}, \xi_{3}, \xi_{2}, \xi_{3}$ i loro valori tratti dalle (11) ed avremo:

$$
\begin{aligned}
& d y_{1}=x \frac{d \cdot \frac{\partial \varphi}{\partial \alpha}+2 d \cdot \frac{\partial \varphi}{\partial \dot{\beta}}}{2 q+\frac{1}{4}}-X d \cdot \frac{\partial \varphi}{\partial \beta} \\
& d y_{2}=y \frac{d \cdot \frac{\partial \varphi}{\partial \alpha}+2 d \cdot \frac{\partial \varphi}{\partial \beta}}{2 q+\frac{1}{4}}-Y d \cdot \frac{\partial \varphi}{\partial \beta} \\
& d y_{3}=z \frac{d \cdot \frac{\partial \varphi}{\partial \alpha}+2 d \cdot \frac{\partial \varphi}{\partial \beta}}{2 q+\frac{1}{4}}-Z d \cdot \frac{\partial \varphi}{\partial \dot{p}} .
\end{aligned}
$$

Poniamo ora :

$$
\psi=-\frac{1}{2} \frac{\varphi}{x+1},
$$

e riguardiamo $\psi$ come funzione di $p, q$ legate per le (11), ad $\alpha, \beta$ dalle re- 
lazioni :

sicchè avremo:

$$
\alpha=\frac{\frac{1}{4}-2 q}{2 q+\frac{1}{4}}, \quad \beta=\frac{p}{2 q+\frac{1}{4}}
$$

$$
\left.\begin{array}{l}
\frac{\partial \dot{\psi}}{\partial p}=-\frac{\partial \varphi}{\partial \beta} \\
\frac{\partial \dot{\psi}}{\partial q}=2(\alpha+1) \frac{\partial \varphi}{\partial \alpha}+2 \alpha \frac{\partial \varphi}{\partial \beta}-2 \rho .
\end{array}\right\}
$$

Le formole (16) possono scriversi in conseguenza così :

$$
\left.\begin{array}{l}
d y_{1}=x d \cdot \frac{\partial \psi}{\partial q}+X d \cdot \frac{\partial \psi}{\partial p} \\
d y_{2}=y d \cdot \frac{\partial \psi}{\partial q}+Y d \cdot \frac{\partial \psi}{\partial p} \\
d y_{3}=z d \cdot \frac{\partial \psi}{\partial q}+Z d \cdot \frac{\partial \Psi}{\partial p} \cdot
\end{array}\right\}
$$

D'altronde, indicando con $r_{1}^{\prime}, r_{2}^{\prime}$ i raggi principali di curvatura della superficie $S^{\prime}$ dello spazio enclideo, immagine della nostra superficie $S$, abbiamo:

$$
\begin{aligned}
\frac{1}{r_{1}}+\frac{1}{r_{2}} & =4 p-\left(2 q+\frac{1}{4}\right)\left(\frac{1}{r_{1}^{\prime}}+\frac{1}{r_{2}^{\prime}}\right) \\
\frac{1}{r_{1} r_{2}} & =\left(2 q+\frac{1}{4}\right)^{2} \frac{1}{r_{1} r_{2}^{\prime}}-2 p\left(2 q+\frac{1}{4}\right)\left(\frac{1}{r_{1}^{\prime}}+\frac{1}{r_{2}^{\prime}}\right)+4 p^{2} .
\end{aligned}
$$

$\mathrm{Ma}$ dalle (19) deduciamo:

$$
\begin{aligned}
\frac{\partial^{2} \psi}{\partial p^{2}} & =-\frac{1}{2 q+\frac{1}{4}} \frac{\partial^{2} \varphi}{\partial \psi^{2}} \\
\frac{\partial^{2} \psi}{\partial p \partial q} & =\frac{1}{\left(2 q+\frac{1}{4}\right)^{2}}\left[\frac{\partial^{2} \varphi}{\partial \alpha \partial \beta}+2 \frac{\partial^{2} \varphi}{\partial \beta^{2}}\right] \\
\frac{\partial^{2} \psi}{\partial q^{2}} & =-\frac{1}{\left(2 q+\frac{1}{4}\right)^{3}}\left[\frac{\partial^{2} \rho}{\partial \alpha^{2}}+4 p \frac{\partial^{2} \varphi}{\partial \alpha \partial \beta}+4 p^{2} \frac{\partial^{2} \varphi}{\partial \beta^{2}}\right]
\end{aligned}
$$

e se nella (13), cui soddisfano $r_{1}, r_{2}$, sostituiamo $i$ valori (21) ed abbiamo 
riguardo alle formole superiori, vediamo che le superficie $S^{\prime}$ soddisfano l'equazione del secondo ordine:

$$
\frac{\partial^{2} \psi}{\partial q^{2}}+\frac{\partial^{2} \psi}{\partial p \partial q}\left(\frac{1}{r_{1}^{\prime}}+\frac{1}{r_{2}}\right)+\frac{\partial^{2} \psi}{\partial p^{2}} \frac{1}{r_{1}^{\prime} r_{2}^{\prime}}=0 .
$$

Le formole (20), (22) coincidono precisamente colle fondamentali dell'ordinario metodo di Weinoartes, onde concludiamo:

La classe di superficie applicabili, dedotte col metodo del $\$ 3$ dalle superficie $S$ dello spazio curvo, che soddisfano la (13), coincide con quella che l'ordinario metodo di WervaArten fa derivare dalle loro superficie immagini dello spazio euclideo, le quali soddisfano alla loro volta l'equazione (22) di Weingarten.

Senza ripetere per lo spazio iperbolico i calcoli analoghi, basterà indicare che il valore della funzione $\psi(p, q)$ che figura in questo caso nell'equazione (22) cui soddisfano le superficie immagini $S^{\prime}$, è dato allora da:

$$
\psi=-\frac{1}{2} \frac{\varphi}{\alpha-1},
$$

essendo attualmente $\alpha, \beta$ legate a $p, q$ dalle formole $\left(11^{*}\right)$ :

$$
\alpha=\frac{2 q+\frac{1}{4}}{2 q-\frac{1}{4}}, \quad \beta=\frac{p}{2 q-\frac{1}{4}} .
$$

\section{$\S 5$.}

\section{Applicazione alle superficie a curvatura assoluta nulla.}

Il metodo di WeINGARTEN, applicato alla geometria ellittica od iperbolica, non dà nulla di nuovo quanto all' effettivo contenuto, come sopra abbiamo dimostrato; l'utilità della trasformazione cos̀̀ conseguita risulterà però evidente dalle applicazioni che ora ci proponiamo di svolgere. In queste o vedremo mostrarsi l'identità di problemi in apparenza ben diversi o stabiliremo nuovi risultati di geometria non euclidea. Cominciamo dall'applicare il metodo del $\S 3$ alle superficie a curvatura assoluta nulla dello spazio ellittico ed jperbolico, a quelle superficie cioè che hanno nel rispettivo spazio curvo

la geometria del piano euclideo. Nel caso ellittico le superficie in discorso 
soddisfano l'equazione :

$$
\frac{1}{r_{1} r_{2}}=-1 \text {, }
$$

che è un caso particolare della (13) ove si ponga:

$$
q=\frac{\alpha^{2}+p^{2}}{2} \text {. }
$$

La classe di ordinarie superficie applicabili dedotta col metodo di WeINGARTEN arrà, secondo la (15), l'elemento lineare:

$$
d s^{2}=\left(1-\alpha^{2}\right) d \alpha^{2}-2 \alpha \beta d \alpha d \beta+\left(1-\beta^{2}\right) d \beta^{2} .
$$

Ponendo :

$$
\alpha=\rho \cos \theta, \quad \beta=\rho \operatorname{sen} \theta,
$$

otteniamo la nota forma :

$$
d s^{2}=\left(1-\rho^{2}\right) d \rho^{2}+\rho^{2} d \theta^{2},
$$

che appartiene alle evolute di quelle superficie $W$ i cui raggi di curvatura sono legati dalla relazione:

$$
r_{1}-r_{2}=\operatorname{sen}\left(r_{1}+r_{2}\right) \text {. }
$$

La classe di superficie d'elemento lineare (24) è interamente nota $\left({ }^{*}\right)$. Da queste superficie, per le quali è dunque :

$$
d y_{1}^{2}+d y_{2}^{2}+d y_{3}^{2}==\left(1-\rho^{2}\right) d \rho^{2}+\rho^{2} d \theta^{2},
$$

possiamo dedurre, secondo il nostro metodo, tutte le superficie a curvatura nulla dello spazio ellittico colle formole seguenti:

$$
\begin{gathered}
x_{0}=\rho \cos \theta, \quad x_{i}=\cos \theta \frac{\partial y_{i}}{\partial \rho}-\frac{\operatorname{sen} \theta}{\rho} \frac{\partial y_{i}}{\partial \theta} \\
(i=1,2,3) \\
\hat{亏}_{0}=\rho \operatorname{sen} \theta, \quad \hat{亏}_{i}=\operatorname{sen} \theta \frac{\partial y_{i}}{\partial \rho}+\frac{\cos \theta}{\rho} \frac{\partial y_{i}}{\partial \theta},
\end{gathered}
$$

che definiscono le coordinate di WeIERstrass $x_{i}$ di un punto mobile della superficie e i coseni di direzione $\xi_{i}$ della normale. Nel caso iperbolico le superficie a curvatura assoluta nulla corrispondono alla relazione:

$$
\frac{1}{r_{1} r_{2}}=1
$$

(*) V. Lezioni, pag. 309. 
e soddisfano alla (13) ove si faccia:

$$
p=\frac{\alpha^{2}-\beta^{2}}{2} .
$$

La classe di superficie applicabili dedotta colle (14) ha per la $\left(15^{*}\right)$ l'elemento lineare:

$$
d s^{2}=\left(\alpha^{2}-1\right) d \alpha^{2}-2 \alpha \beta d \alpha d \beta+\left(1+\beta^{2}\right) d \beta^{2},
$$

che, ponendo :

$$
\alpha=\rho \cosh \theta, \quad \beta=\rho \operatorname{senh} \theta,
$$

diventa :

$$
d s^{2}=\left(\rho^{2}-1\right) d \rho^{2}+\rho^{2} d \sigma^{2} .
$$

Questo elemento lineare appartiene alle complementari delle superficie applicabili sul paraboloide di rotazione $\left({ }^{*}\right)$ ed anche qui è nota tutta la classe di queste superficie applicabili. Da esse deduciamo le superficie a curvatura nulla dello spazio iperbolico colle formole:

$$
\begin{aligned}
& x_{0}=\rho \cosh \theta, \quad x_{i}=\cosh \theta \frac{\partial y_{i}}{\partial i}-\frac{\operatorname{senh} \theta}{i} \frac{\partial y_{i}}{\partial i} \\
& \xi_{0}=\rho \operatorname{senh} \theta, \quad \xi_{i}=\operatorname{senh} \theta \frac{\partial y_{i}}{\partial \rho}-\frac{\cosh \theta}{i} \frac{\partial y_{i}}{\partial \theta} .
\end{aligned}
$$

Che le superficie a curvatura nulla degli spazi a curvatura costante potessero tutte ottenersi in termini finiti avevo già altrove stabilito (**). Ma qui la loro determinazione appare sotto nuovo aspetto collegandosi al problema già risoluto di trovare le ordinarie superficie d'elemento lineare (24) o $\left(24^{*}\right)$.

(*) V. Lezioni, pag. 312 .

(*) V. la mia Nota: Sulle superficie a curvatura mulla negli spazi a curvalura costante (Atti dell'Accademia di Torino, 9 giugno 1895̆) e la successiva Memoria nel tom. XXIV degli Annali di Matematica. 


\section{$\S 6$.}

\section{Le superficie d'area minima e le superficie di LiouviLLE derivate.}

La ricerca delle superficie d'area minima in geometria ellittica od iperbolica dipende, come già dimostrai nella mia Memoria del 1838 (Atti dei Lincei) $\left(^{*}\right)$, dalla integrazione dell'equazione a derivate parziali :

$$
\frac{\partial^{2} \theta}{\partial u^{2}}+\frac{\partial^{2} \theta}{\partial v^{2}}=-2 \operatorname{senh} 26
$$

per 10. spazio ellittico e dall'altra:

$$
\frac{\partial^{2} \theta}{\partial u^{2}}+\frac{\partial^{2} \theta}{\partial v^{2}}=2 \cosh 2 \theta
$$

per lo spazio iperbolico. La prima coincide con quella da cui dipende la ricerca delle superficie a curvatura costante positiva (o a curvatura media costante) dello spazio euclideo.

Se applichiamo il metodo di Weingarten ( $\$ 3$ ) alle superficie minime, dovremo fare evidentemente:

$$
\varphi=\alpha \beta .
$$

Per l'elemento lineare delle superficie derivate otterremo quindi nel caso ellittico :

$$
d s^{2}=\left(1-\beta^{2}\right) d \alpha^{2}-2 \alpha \beta d \alpha d \beta+\left(1-\alpha^{2}\right) d \beta^{2},
$$

e nel caso iperbolico:

$$
d s^{2}=\left(1+\beta^{2}\right) d \alpha^{2}+2 \alpha \beta d \alpha d \beta+\left(\alpha^{2}-1\right) d \beta^{2} .
$$

L'uno e l'altro elemento lineare appartengono alla forma di Lrouvmu. Pongasi invero per la (26):

$$
\alpha=\operatorname{sen} \frac{u+v}{2}, \quad \beta=\operatorname{sen} \frac{u-v}{2},
$$

e risulterà :

$$
d s^{2}=\frac{1}{4}(\cos u+\cos v)\left(\cos u d u^{2}+\cos v d v^{2}\right)
$$

(*) Cf. anche Darboux, Legons, tom. III, pag. 171 . 
che ha appunto la forma di Liouvine. Nell'altro caso poniamo invece:

$$
\alpha=\cosh \frac{u+v}{2}, \quad \beta=\operatorname{senh} \frac{u-v}{2},
$$

e la $\left(26^{*}\right)$ diventa :

$$
d s^{2}=\frac{1}{4}(\operatorname{senh} u+\operatorname{senh} v)\left(\operatorname{senh} u d u^{2}+\operatorname{senh} v d v^{2}\right)
$$

Così vediamo che sono due problemi equivalenti la ricerca delle superficie a curvatura costante positiva o quella delle superficie d'elemento lineare (27). Come tipo di questa classe possiamo prendere ad esempio quella superficie che deriva dalla superficie d'area minima doppiamente rigata di Cujfrord, definita dalle formole:

$$
\begin{aligned}
& x_{0}=\cos u \cos v, \quad x_{1}=\operatorname{sen} u \cos v, \quad x_{2}=\cos u \operatorname{sen} v, \quad x_{3}=\operatorname{sen} u \operatorname{sen} v, \\
& \xi_{0}=\operatorname{sen} u \operatorname{sen} v, \quad \xi_{1}=-\cos u \operatorname{sen} v, \quad \xi_{2}=-\operatorname{sen} u \cos v, \quad \xi_{3}=\cos u \cos v,
\end{aligned}
$$

dove $u, v$ sono i parametri delle rette (assintotiche). Per la superficie di LuouviLhe derivata avremo:

$$
d y_{i}=x_{i} d \xi_{0}+\xi_{i} d x_{0}, \quad(i=1,2,3),
$$

ed eseguendo le quadrature:

$$
\begin{gathered}
y_{1}=\frac{1}{4}(2 v-\cos 2 u \operatorname{sen} 2 v), \quad y_{2}=\frac{1}{4}(2 u-\operatorname{sen} 2 u \cos 2 v), \\
y_{3}=\frac{1}{4} \cos 2 u \cos 2 v .
\end{gathered}
$$

Sostituendovi una superficie omotetica e girando gli assi coordinati $x, y$ di $45^{\circ}$, possiamo scrivere:

$$
x=\frac{U-\operatorname{sen} U}{\sqrt{2}}, \quad y=\frac{V-\operatorname{sen} V}{\sqrt{2}}, \quad z=\frac{1}{2}[\cos U+\cos V] .
$$

Queste formole definiscono una superficie di traslazione, le cui due curve generatrici nei piani perpendicolari $x z, y z$ sono identiche; essa è nello stesso tempo una superficie di Lrouvilue d'elemento lineare proporzionale a (27). 


\section{$\S 7$.}

\section{Le superficie d'APPELL-BoNNET in geometria ellittica.}

Nello spazio euclideo diconsi superficie di Bonser quelle per le quali i punti medi fra i centri principali di curvatura, sopra ogni normale, giacciono in un piano; superficie d'APPELl quelle i cui piani medi, cioè i piani perpendicolari nei punti medi alle normali della superficie, concorrono in un punto $\left({ }^{*}\right)$. Se prendiamo ora a considerare le superficie analoghe in geometria ellittica, facilmente vediamo che, a causa della perfetta legge di dualità vigente nello spazio ellittico, ogni superficie d'Appers è altresì una superficie di BonNET e viceversa. Qui in vero sopra ogni normale, che è una retta chiusa di lunghezza $=\pi$, abbiamo due punti medi distanti fra loro di un quadrante e, se i punti medi di una serie giacciono in un piano, i piani medi dell'altra serie passano per un punto, il polo di questo piano.

Poniamo che il piano luogo dei punti medi nella nostra superficie di Bonnet-Appell sia il piano $x_{0}=0$. Indicando con $r_{1}, r_{2}$ i raggi ridotti di curvatura e ponendo:

$$
r_{1}=\operatorname{tang} w_{1}, \quad r_{2}=\operatorname{tang} w_{2},
$$

la nostra superficie sarà caratterizzata dalla relazione:

$$
x_{0} \cos \frac{w_{1}+w_{2}}{2}-\xi_{0} \operatorname{sen} \frac{w_{1}+w_{2}}{2}=0,
$$

ovvero:

$$
\operatorname{tang} \frac{w_{1}+w_{2}}{2}=\frac{\alpha}{\beta} .
$$

Se ne ricava:

$$
\operatorname{tang}\left(w_{1}+w_{2}\right)=\frac{2 \alpha \beta}{\beta^{2}-\alpha^{2}},
$$

ovvero:

$$
\alpha \beta r_{1} r_{2}+\frac{1}{2}\left(\beta^{2}-\alpha^{2}\right)\left(r_{1}+r_{2}\right)-\alpha \beta=0 .
$$

Questa equazione può porsi sotto la forma (13) $\S 3$, dando alla funzione $\varphi$

(*) Cf. Lezioni, pag. 231 e 291 . 
il valore :

$$
\varphi=\operatorname{arctg} \frac{\beta}{\alpha} .
$$

Ora, calcolando dalla (15) $\S 3$ l'elemento lineare della classe di superficie applicabili derivate secondo il metodo di Weingarten, troviamo:

e ponendo:

$$
d s=\frac{\left(1-\beta^{2}\right) d \alpha^{2}+2 \alpha \beta d \alpha d \beta+\left(1-\alpha^{2}\right) d \beta^{2}}{\left(\alpha^{2}+\beta^{2}\right)^{2}},
$$

abbiamo anche:

$$
\alpha=\frac{\cos \theta}{p}, \beta=\frac{\operatorname{sen} \theta}{?},
$$

$$
d s^{2}=d \rho^{2}+\left(\rho^{2}-1\right) d \theta^{2} \text {. }
$$

Questa forma dell'elemento lineare appartiene alle evolute delle superficie a curvatura costante positiva e però vediamo che: La ricerca delle superficie di Bonnet-APPelu nello spazio ellittico equivale a quella delle superficie a curvatura costante positiva dello spazio euclideo. Risultati analoghi potremmo dedurre per le superficie di APPELl e di BonNet nello spazio iperbolico.

\section{$\S 8$.}

Le superficie dello spazio iperbolico per le quali $\frac{1}{r_{1}}+\frac{1}{r_{2}}=2$

$$
\text { o } r_{1}+r_{2}=2 \text {. }
$$

Volgiamoci ora alla principale applicazione che abbiamo in vista, considerando quelle superficie dello spazio iperbolico a curvatura $K=-1$, per le quali la somma dei raggi principali di curvatura ridotti o delle loro inverse è eguale a 2 .

Secondo le formole fondamentali (8), (9) del $\S 1$, esiste una superficie dello spazio iperbolico, il cui elemento lineare, riferito alle linee di curvatura $u, v$, ̀̀ dato da:

$$
d s^{2}=e^{2 \theta}\left(d u^{2}+d v^{2}\right)
$$

e le curvature principali da:

$$
\frac{1}{r_{1}}=1-e^{-2 \theta}, \quad \frac{1}{r_{2}}=1+e^{-2 \theta},
$$


purchè $\theta$ soddisfi alla equazione (9) di Gauss, che qut assume la forma :

$$
\frac{\partial^{2} \theta}{\partial u^{2}}+\frac{\partial^{2} \theta}{\partial v^{2}}=e^{-2 \theta} \text {. }
$$

Di questa equazione (di Lrouritre) si conosce l'integrale generale e ad ogni sua soluzione corrisponde dunque una superficie dello spazio iperbolico colla curvatura media costante:

$$
\frac{1}{r_{1}}+\frac{1}{r_{2}}=2
$$

Similmente, se facciamo:

$$
\begin{gathered}
d s^{2}=4\left(\operatorname{senh}^{2} \theta d u^{2}+\cosh ^{2} \theta d v^{2}\right), \\
r_{1}=1+e^{2 \theta}, \quad r_{2}=1-e^{2 \theta},
\end{gathered}
$$

sono soddisfatte le equazioni (8) di Codazzs, e quella (9) di Gauss diventa :

$$
\frac{\partial^{2} \theta}{\partial u^{2}}+\frac{\partial^{2} \theta}{\partial v^{2}}=e^{2 \theta} \text {. }
$$

Ad ogni soluzione di questa equazione, di cui è pur noto l'integrale generale $(*)$, corrisponderà una superficie dello spazio jperbolico per la quale sarà :

$$
r_{1}+r_{2}=2 \text {, }
$$

ed inversamente. Così se applichiamo il metodo generale del $\S 1$ che serve per risalire dalle due forme fondamentali date alla corrispondente superficie, il problema di determinare nello spazio iperbolico le nostre superficie:

$$
\frac{1}{r_{1}}+\frac{1}{r_{2}}=2, \quad r_{1}+r_{2}=2,
$$

appare ridotto alla integrazione, che sappiamo effettuare, delle (28), (30) e a quella successiva del corrispondente sistema di equazioni differenziali (6), (7).

(*) L'integrazione completa delle (28), (30) puo presentarsi sotto un aspetto geometrico ben semplice. $E$ infatti la forma differenziale quadratica:

$$
e^{-2 \theta}\left(d u^{2}+d v^{2}\right)
$$

se $\theta$ soddisfa la (28), ha la curvatura $k=1$ e l'altra:

$$
e^{2 \theta}\left(d u^{2}+d v^{2}\right)
$$

quando $\theta$ sia una soluzione della (30), ha la curvatura $k=-1$. La integrazione delle (28), (30) equivale quindi alla ricerca dei sistemi isotermi sulla sfera o sulla pseudosfera, problema di ben nota risoluzione. 
Ma ora, applicando a queste superficie il metodo di Wengarten, potremo spingere ben più in là la ricerca ed ottenere in termini finiti tutte le superficie delle due classi.

\section{$\S 9$.}

\section{Applicazione del metodo di Wengarten alle superficie del paragrafo precedente.}

Le superficie dello spazio jperbolico per le quali :

$$
\frac{1}{r_{1}}+\frac{1}{r_{2}}=2,
$$

soddisfano all'equazione fondamentale (13) $\S 3$, ove si faccia :

similmente le altre :

$$
\varphi=\alpha \beta-\alpha^{2}
$$

assumendo invece :

$$
r_{1}+r_{2}=2
$$

$$
\varphi=\alpha \beta-\beta^{2} .
$$

Ora se applichiamo il metodo di Weingarten (\$3) per dedurne due classi complete di superficie applicabili dello spazio ordinario, troviamo nel primo caso:

$d s^{2}=\left[(2 \alpha-\beta)^{2}-3\right] d \alpha^{2}+2\left[\alpha \beta-2\left(\alpha^{2}-1\right)\right] d \alpha d \beta+\left(\alpha^{2}-1\right) d \beta^{2}$,

e nel secondo:

$d s_{1}^{2}=\left(1+\beta^{2}\right) d \alpha^{2}+2\left[\alpha \beta-2\left(1+\beta^{2}\right)\right] d \alpha d \beta+\left[(\alpha-2 \beta)^{2}+3\right] d \beta^{2} . \quad\left(32^{*}\right)$

Possiamo ridurre questi due elementi lineari a forme ben note medianto le sostituzioni seguenti. Pongasi nella (32):

$$
v=\log (\alpha-\beta), \quad 2 u=2 \alpha^{2}-2 \alpha \beta-2 \log (\alpha-\beta)-1,
$$

e si otterrà la forma equivalente:

$$
d s^{2}=d u^{2}+\left(2 u+2 v-e^{2 v}\right) d v^{2} .
$$

Nella (32*) pongasi invece:

$$
v=\log (\alpha-\beta), \quad 2 u=2 \alpha \beta-2 \beta^{2}-2 \log (\alpha-\beta)-1,
$$


e si otterrà :

$$
d s_{1}^{2}=a^{2} u^{2}+\left(2 u+2 v+e^{2 v}\right) d v^{2} .
$$

Ora lo ultime ricerche di Weingarten (come si vedrà riportato nel paragrafo seguente) dànno il modo di trovare in termini finiti tutte le ordinarie superficie di elemento lineare (34) o $\left(34^{*}\right)$ e da queste potremo ottenere con sole derivazioni, nel modo che andiamo ad indicare, le superficie dello spazio iperbolico:

$$
\frac{1}{r_{1}}+\frac{1}{r_{2}}=2, \quad r_{1}+r_{2}=2 .
$$

Suppongasi per ciò di avere una individuata superficie $\Sigma$ della classe (34), per la quale adunque si abbia:

$$
d y_{1}^{2}+d y_{2}^{2}+d y_{3}^{2}=d u^{2}+\left(2 u+2 v-e^{2 v}\right) d v^{2} .
$$

Se teniamo conto delle formole del $\S 3$ e delle attuali formole di trasformazione (33) pel passaggio delle coordinate $\alpha, \beta$ alle $u, v$ troviamo che la corrispondente superficie $S$ a curvatura media costante $=2$ è definita dalle formole:

$$
\begin{aligned}
& x_{0}=\left(\frac{1}{2}+u+v\right) e^{-v} \\
& x_{i}=\left(\frac{1}{2}-u-v\right) e^{-v} \frac{\partial y_{i}}{\partial u}-e^{-v} \frac{\partial y_{i}}{\partial v} \quad(i=1,2,3), \\
& 亏_{0}=\left(\frac{1}{2}+u+v\right) e^{-v}-e^{v} \\
& 亏_{i}=\left[\left(\frac{1}{2}-u=v\right) e^{-v}+e^{v}\right] \frac{\partial y_{i}}{\partial u}-e^{-v} \frac{\partial y_{i}}{\partial v} \quad(i=1,2,3) .
\end{aligned}
$$

Del tutto similmente, avendosi una superficie $\Sigma$ d'elemento lineare:

$$
d y_{1}^{2}+d y_{2}^{2}+d y_{3}^{2}=d u^{2}+\left(2 u+2 v+e^{2 v}\right) d v^{2},
$$

definiremo la corrispondente superficie $S$ dello spazio iperbolico con $r_{1}+r_{3}=2$ mediante le formole:

$$
\begin{aligned}
& x_{0}=\left(u+v+\frac{1}{2}\right) e^{-v}+e^{v} \\
& x_{i}=\left[\left(u+v-\frac{1}{2}\right) e^{-v}+e^{v}\right] \frac{\partial y_{i}}{\partial u}+e^{-v} \frac{\partial y_{i}}{\partial v} \quad(i=1,2,3), \\
& \xi_{0}=\left(u+v+\frac{1}{2}\right) e^{-v} \\
& \xi_{i}=\left(u+v-\frac{1}{2}\right) e^{-v} \frac{\partial y_{i}}{\partial u}+e^{-v} \frac{\partial y i}{\partial v} \quad(i=1,2,3) .
\end{aligned}
$$


$\grave{\mathrm{E}}$ ben facile procedere ora ad una verifica delle formole precedenti. In particolare, supposto the $\Sigma$ sia una superficie d'elemento lineare (35), verifichiamo che la superficie $S$ dello spazio iperbolico definita dalle (36), avrà la curvatura media:

E invero avendosi:

$$
\frac{1}{r_{1}}+\frac{1}{r_{2}}=2 \text {. }
$$

$$
\mathbf{\Sigma}\left(\frac{\partial y_{i}}{\partial u}\right)^{2}=1, \quad \mathbf{y} \frac{\partial y_{i}}{\partial u} \frac{\partial y_{i}}{\partial v}=0, \quad \mathbf{y}\left(\frac{\partial y_{i}}{\partial v}\right)^{2}=2 u+2 v-e^{2 v},
$$

si verificano subito le relazioni:

$$
\begin{gathered}
\sum_{i} x_{i}^{2}-x_{0}^{2}=-1, \quad \sum_{i} \xi_{i} x_{i}-\xi_{0} x_{0}=0, \quad \sum_{i} \xi_{i}^{2}-\xi_{0}^{2}=1, \\
\sum_{i} \xi_{i} \frac{\partial x_{i}}{\partial u}-\xi_{0} \frac{\partial x_{0}}{\partial u}=0, \quad \sum_{i} \xi_{i} \frac{\partial x_{i}}{\partial v}-\xi_{0} \frac{\partial x_{0}}{\partial v}=0,
\end{gathered}
$$

cio che dimostra intanto che per la detta superficie $S$ le (37) dànno effettivamente le coordinate del piano tangente. Se osserviamo poi che spostandoci lungo una linea di curvatura della $S$ dobbiamo avere:

$$
\left.\begin{array}{l}
\frac{\partial x_{0}}{\partial u} d u+\frac{\partial x_{0}}{\partial v} d v=r\left(\frac{\partial \xi_{0}}{\partial u} d u+\frac{\partial \xi_{0}}{\partial v} d v\right), \\
\frac{\partial x_{i}}{\partial u} d u+\frac{\partial x_{i}}{\partial v} d v=r\left(\frac{\partial \xi_{i}}{\partial u} d u+\frac{\partial \xi_{i}}{\partial v} d v\right) \quad(i=1,2,3),
\end{array}\right\}
$$

indicando con $r$ il raggio principale ridotto di curvatura, dalle tre ultime moltiplicate ordinatamente per $\mathrm{i}$ coseni di direzione:

$$
Y_{1}, Y_{2}, Y_{3}
$$

della normale alla $\Sigma$ e sommate deduciamo:

$$
\begin{gathered}
{\left[\left(\frac{1}{2}-u-v\right) D-D^{\prime}\right] d u+\left[\left(\frac{1}{2}-u-v\right) D^{\prime}-D^{\prime \prime}\right] d v=} \\
\left.=r^{\prime}\left[\left(\frac{1}{2}-u-v\right) D-D^{\prime}+e^{2 v} D\right] d u+\left[\left(\frac{1}{2}-u-v\right) D^{\prime}-D^{\prime \prime}+e^{2 v} D^{\prime}\right]\right\} d v
\end{gathered}
$$

dove $D, D^{\prime}, D^{\prime \prime}$ indicano i coefficienti della seconda forma fondamentale della superficie $\Sigma$. La prima delle (38) ci dà poi:

$$
d u+\left(\frac{1}{2}-u-v\right) d v=r\left\{d u+\left(\frac{1}{2}-u-v-e^{2 v}\right) d v\right\} .
$$


Eliminando fra questa e la precedente i differenziali $d u, d v$, otteniamo l'equazione di $2 .^{\circ}$ grado in $r$ :

$$
\begin{array}{cl}
{\left[\left(\frac{1}{2}-u-v\right) D-D^{\prime}\right](1-r)+e^{2 v} D \cdot r,} & {\left[\left(\frac{1}{2}-u-v\right) D^{\prime}-D^{\prime \prime}\right](1-r)+e^{2 v} D^{\prime} \cdot r} \\
1-r & \left(\frac{1}{2}-u-v\right)(1-r)-e^{2 v} r
\end{array}
$$

le cui radici $r_{1}, r_{2}$ sono i raggi principali di curvatura di $S$ e fra queste radici ha luogo appunto la relazione:

$$
\frac{1}{r_{1}}+\frac{1}{r_{2}}=2 .
$$

In modo del tutto simile si possono eseguire le verifiche relative alle formole $\left(35^{*}\right),\left(36^{*}\right),\left(37^{*}\right)$.

$\S 10$.

\section{Le superficie d'elemento lineare $d s^{2}=d u^{2}+\left(2 u+2 v \mp e^{i v}\right) d v^{z}$.}

Per raccogliere nella presente Memoria tutte le formole necessarie alla determinazione delle superficie dello spazio iperbolico con:

$$
\frac{1}{r_{1}}+\frac{1}{r_{2}}=2, \quad 0 \quad r_{1}+r_{2}=2,
$$

resta che ricordiamo come si trovano, secondo WeIngarten, tutte le superficie d'elemento lineare $(34)$ o $\left(34^{*}\right)\left({ }^{*}\right)$.

Nel metodo di Weingarten, conoscendo le superficie che soddisfano l'equazione fondamentale $(A)$ :

colle quadrature:

$$
\frac{\partial^{2} \varphi}{\partial p^{2}}+\left(r_{1}+r_{2}\right) \frac{\partial^{2} \varphi}{\partial p \partial q}+r_{1} r_{2} \frac{\partial^{2} \varphi}{\partial q^{2}}=0
$$

$$
\begin{aligned}
& d y_{1}=x d \cdot \frac{\partial \varphi}{\partial q}+X d \cdot \frac{\partial \varphi}{\partial p} \\
& d y_{2}=y d \cdot \frac{\partial \varphi}{\partial q}+Y d \cdot \frac{\partial \varphi}{\partial p} \\
& d y_{3}=z d \cdot \frac{\partial \varphi}{\partial q}+Z d \cdot \frac{\partial \varphi}{\partial p}
\end{aligned}
$$

(*) Or. DArвoux, Lecons, tom. IV, pag. 332. 
si deducono le superficie d'elemento lineare:

$$
d s^{2}=\sum_{i} d y_{i}^{2}=2 q\left(d \frac{\partial \varphi}{\partial q}\right)^{2}+2 p d \cdot \frac{\partial \varphi}{\partial p} d \cdot \frac{\partial}{\partial} \frac{\partial}{q}+\left(d \cdot \frac{\partial \varphi}{\partial p}\right)^{2} .
$$

Facciamo nella $(A)$ :

$$
\varphi=p q-\frac{p^{3}}{3}-\psi(p)
$$

essendo $\psi$ una funzione della sola $p$. L'elemento lineare della superficie derivata, ponendo:

diventa:

$$
u=q-\frac{p^{2}}{2}-\psi^{\prime}(p)
$$

$$
d s^{2}=d u^{2}+\left[2 u+2 \psi^{\prime}(p)\right] d p^{2},
$$

mentre la $(A)$ assume la forma:

Ora se prendiamo:

$$
r_{1}+r_{2}=2 p+\psi^{\prime \prime}(p)
$$

la (40) diventa:

$$
\psi^{\prime}(p)=p \mp \frac{1}{2} e^{2 p}
$$

$$
r_{1}+r_{2}=2 p+1 \mp e^{2 p}
$$

e l'elemento lineare delle superficie derivate assume la forma:

$$
d s^{2}=d u^{2}+\left(2 u+2 p \mp e^{2 p}\right) d p^{2},
$$

che è precisamente quella della classe di superficie da noi ricercata. Resta dunque che vediamo come si determinano tutte le superficie della classe $\left(40^{*}\right)$. Adoperando qui le coordinate tangenziali (*) e ricordando che $p$ denota la distanza dell'origine dal piano tangente, vediamo che l'equazione $\left(40^{*}\right)$ da integrarsi equivale alla seguente:

$$
\Delta_{\mathbf{2}} W=1 \mp e^{2 W},
$$

il parametro differenziale secondo della funzione incognita $W$ essendo calcolato rispetto all'elemento lineare della sfera rappresentativa di Gauss. Assumiamo su questa sfera a linee coordinate $i$ meridiani ed i paralleli, ridotti ai parametri isometrici $u, v$ col porre:

$$
X=\frac{\cos v}{\cosh u}, \quad Y=\frac{\operatorname{sen} v}{\cosh u}, \quad Z=\operatorname{tang} h u,
$$

(*) Cfr. Lezioni, n. 7*, pay. 137, formola (37). 
sicchè :

$$
d s^{\prime 2}=d X^{2}+d Y^{2}+d Z^{2}=\frac{1}{\cosh ^{2} u}\left(d u^{2}+d v^{2}\right),
$$

e la (41) diventerà :

$$
\cosh ^{2} u\left(\frac{\partial^{2} W}{\partial \cdot u^{2}}+\frac{\partial^{2} W}{\partial v^{2}}\right)=1 \mp e^{2 W}
$$

Poniamo ora:

$$
W=\log \cos h u+\theta
$$

e per la nuova funzione incognita $\theta$ avremo precisamente l'equazione di LIOUVILLE:

$$
\frac{\partial^{2} \theta}{\partial u^{2}}+\frac{\partial^{2} \theta}{\partial v^{2}}=\mp e^{2 \theta}
$$

Da ogni soluzione di questa equazione deduciamo, secondo le formole in coordinate tangenziali, con sole derivazioni, le corrispondenti superficie d'elemento lineare:

$$
d s^{2}=d u^{2}+\left(2 u+2 v \mp e^{2 v}\right) d v^{2},
$$

indi colle $(36),\left(36^{*}\right)$ del $\S 9$ le superficie dello spazio iperbolico che soddisfano le equazioni:

$$
\frac{1}{r_{1}}+\frac{1}{r_{2}}=2, \quad 0 \quad r_{1}+r_{2}=2 .
$$

Così vediamo nuovamente (cf. $§ 8$ ) che la determinazione di queste superficie dipende dalla equazione (43) di LiourmLe; ma questa volta il risultato viene completato nel senso che colla integrazione della (43) otteniamo altresi in termini finiti le superficie richieste.

\section{$\S 11$.}

\section{Rappresentazione sferica di GaUss nello spazio iperbolico.}

Per stabilire la proprietà caratteristica delle superficie a curvatura media :

$$
\frac{1}{r_{1}}+\frac{1}{r_{2}}=2,
$$

dello spazio iperbolico, come l'abbiamo enunciata nella prefazione, convieve prima che diciamo brevemente delle proprietà generali della rappresentazione 
sferica di Gauss in geometria non-euclidea. Ricordiamo per sio che ad una retta arbitraria dello spazio iperbolico partono da ogni punto due distinte parallele. Ora, presa una superficie qualsiasi $S$ ed un punto fisso $O$ dello spazio, conduciamo per $O$ i due raggi paralleli ad ogni singola normale di $S$ ed intersechiamo questo doppio sistema di raggi con una sfera $\Sigma$ di contro $O$. Otteniamo cosi due rappresentazioni di $S$ sulle sfera $\Sigma$, she sono da considerarsi come le analoghe della rappresentazione sferica di Gauss nello spazio euclideo. Una qualunque di queste due rappresentazioni sferiche gode, come ora vedremo, della proprietà fondamentale della rappresentazione di Gıuss e cioè: Il sistema ortogonale delle linee di curvatura di S ha nell'immagine sferica una raporesentazione ortogonale.

In generale è questo il solo sistema ortogonale di $S$ che si conserva ortogonale nella rappresentazione, a meno che la rappresentazione riesca conforme: ciò accade soltanto per le sfere e per le superficie a curvatura media costante:

$$
\frac{1}{r_{1}}+\frac{1}{r_{2}}=\mp 2 \text {. }
$$

Queste ultime superficie vengono cosi caratterizzate dalla proprietà enunclata nella prefazione e si ravvicinano per tal modo alle superficie minime dello spazio euclideo.

Per dimostrare Je asserzioni superiori, cominciamo dal ricordare che una retta nello spazio iperbolico viene fissata, in coordinate di Weierstrass, dando le coordinate:

$$
x_{0}, x_{1}, x_{2}, x_{3}
$$

di un suo punto e le coordinate:

$$
\xi_{0}, \xi_{1}, \xi_{2}, \xi_{3},
$$

del piano normale alla retta nel punto $\left(x_{i}\right)$; le $x$, relazioni:

$$
\sum_{i} x_{i}^{2}-x_{0}^{2}=-1, \quad \sum_{i} \xi_{i}^{2}-\xi_{0}^{2}=1, \quad \sum_{i} \xi_{i} x_{i}-\xi_{0} x_{0}=0
$$

Prendiamo per punto fisso dello spazio, dal quale vogliamo condurre le due parallele alla retta il punto $O \equiv(1,0,0,0)$ e indicando con $\overline{\xi_{0}}, \overline{\bar{\zeta}_{1}}, \overline{\bar{\xi}_{2}}, \overline{\xi_{3}}$ le coordinate del piano per $O$ normale all'una o all'altra delle due parallele condotte per $O$ alla detta retta $\left(x_{i}, \xi_{i}\right)$ troveremo mediante considerazioni 
elementari $\left(^{*}\right)$ :

$$
\bar{\xi}_{0}=0, \quad \bar{\zeta}_{1}=\frac{x_{1} \pm \bar{\xi}_{1}}{x_{0} \pm \bar{\xi}_{0}}, \quad \bar{\xi}_{2}=\frac{x_{2} \pm \bar{\zeta}_{2}}{x_{0} \pm \bar{\zeta}_{0}}, \quad \bar{\xi}_{3}=\frac{x_{3} \pm \xi_{3}}{x_{0} \pm \xi_{0}},
$$

l'una delle due parallele corrispondendo ai segni superiori, l'altra agli inferiori. Per fissare le idee scegliamo i segni superiori e poniamo:

$$
\vec{\xi}_{0}=0, \quad \overline{\xi_{i}}=\frac{x_{i}+\bar{\xi}_{i}}{x_{0}+\bar{\xi}_{0}} \quad(i=1,2,3) \text {. }
$$

Riferiamo la superficie $S$ di cui vogliamo studiare la rappresentazione sferica alle sue linee di curvatura $(u, v)$ talchè, indicando con $x_{i}$ le coordinate di un punto di $S_{i}$ con $\xi_{i}$ quelle del piano tangente, avremo:

$$
\frac{\partial x_{i}}{\partial u}=r_{2} \frac{\partial \zeta_{i}}{\partial u}, \quad \frac{\partial x_{i}}{\partial v}=r_{1} \frac{\partial \zeta_{i}}{\partial v} \quad(i=0,1,2,3) \text {. }
$$

(*) Per stabilire le tormole del testo si cominci dall'osservare che se si hanno due punti :

$$
\left(x_{0}^{\prime} x_{1}^{\prime} x_{2}^{\prime} x_{3}^{\prime}\right), \quad\left(\bar{x}_{0} \bar{x}_{1} \bar{x}_{2} \bar{x}_{3}\right)
$$

e si considera la loro congiungente, le coordinate $\bar{\xi}_{i}$ del piano normale nel punto $\left(x^{\prime}\right)$ a questa congiungente sono date $\mathrm{da}$ :

$$
\bar{\xi}_{i}=\frac{\bar{x}_{i}}{\sqrt{\left[S\left(x^{\prime} x\right)\right]^{2}-1}}-\frac{x_{i}^{\prime} \cdot S\left(x^{\prime} x\right)}{\sqrt{\left(S x^{\prime} x\right)^{2}-1}}
$$

dove si è posto:

$$
S\left(x^{\prime} x\right)=x_{0}^{\prime} \bar{x}_{0}-\sum_{i} w_{i}^{\prime}, \bar{x}_{i} \text {. }
$$

Ora sulla retta $(x, \xi)$ prendiamo un punto $(\bar{x})$ alla distanza $w$ da $(x)$; avremo:

$$
\overline{x_{i}}=x_{i} \cosh w+\xi_{i} \operatorname{senh} w,
$$

e per la congiungente di due punti $\left(x^{\prime}\right)(\bar{x})$ varranno le formole $(x)$. Facciamo ora crescere 20 all'infinito positivo o negativo osservando che:

a quindi :

$$
\lim _{w= \pm \infty} \frac{x_{i}}{\cosh w}=x_{i} \pm \xi_{i}
$$

$$
\lim _{w= \pm \infty} \frac{S\left(x^{\prime} \bar{x}\right)}{\cosh w}=S\left(x^{\prime} x\right) \pm S\left(\xi x^{\prime},\right.
$$

e le formole ( $($ ) diventano:

$$
\overline{\xi_{i}}=\frac{x_{i} \pm \xi_{i}}{S(x x) \pm S\left(\xi x^{\prime}\right)}-x_{i}^{\prime},
$$

che per $x_{0}^{\prime}=1, x_{1}^{\prime}=x_{2}^{\prime}=x_{3}^{\prime}=0$ dànno le formole del testo. 
Dalle (44), (45) deduciamo derivando:

$$
\begin{aligned}
& \frac{\partial \bar{\xi}_{i}}{\partial u}=\left(1+\frac{1}{r_{2}}\right) \frac{\left(x_{0}+\xi_{0}\right) \frac{\partial x_{i}}{\partial u}-\left(x_{i}+\xi_{i}\right) \frac{\partial x_{0}}{\partial u}}{\left(x_{0}+\xi_{0}\right)^{2}} \\
& \frac{\partial \overline{\xi_{i}}}{\partial v}=\left(1+\frac{1}{r_{1}}\right) \frac{\left(x_{0}+\xi_{0}\right) \frac{\partial x_{i}}{\partial v}-\left(x_{i}+\xi_{i}\right) \frac{\partial x_{0}}{\partial v}}{\left(x_{0}+\xi_{0}\right)^{2}},
\end{aligned}
$$

e quindi ponendo:

troveremo $\left(^{*}\right)$ :

$$
d s_{1}^{2}=d \overline{\xi_{1}^{2}}+d \overline{\xi_{2}^{2}}+d \overline{\xi_{3}^{2}}
$$

$$
d s_{1}^{2}=\frac{E\left(1+\frac{1}{r_{2}}\right)^{2} d u^{2}+G\left(1+\frac{1}{r_{1}}\right)^{2} d v^{2}}{\left(x_{0}+\xi_{0}\right)^{2}} \text {. }
$$

Ora $d s_{1}^{2}$ non differisce che per un fattore costante dal quadrato dell'elemento lineare della sfera rappresentativa, sulla quale adunque le immagini $u, v$ delle linee di curvatura di $S$ costituiscono, come si era asserito, un sistema ortogonale. Nessun altro sistema ortogonale di $S$ si conserva ortogonale nella rappresentazione a meno che non si abbia rappresentazione conforme, il che accade quando:

ciò̀:

$$
\frac{E\left(1+\frac{1}{r_{z}}\right)^{2}}{E}=\frac{G\left(1+\frac{1}{r_{1}}\right)^{2}}{G}
$$

$$
1+\frac{1}{r_{2}}= \pm\left(1+\frac{1}{r_{1}}\right)
$$

Sarà dunque o $r_{1}=r_{2}$, nel qual caso $S$ è una sfera, ovvero:

$$
\frac{1}{r_{1}}+\frac{1}{r_{2}}=-2\left({ }^{* *}\right)
$$

ciò che dimostra tutte le nostre asserzioni.

(*) Si tengano presenti le relazioni:

$$
\begin{array}{ll}
\sum_{i}\left(\frac{\partial x_{i}}{\partial u}\right)^{2}=E+\left(\frac{\partial x_{0}}{\partial u}\right)^{2}, & \underset{i}{\Sigma} \frac{\partial x_{i}}{\partial u} \frac{\partial x_{i}}{\partial v}=\frac{\partial \nu_{0}}{\partial u} \frac{\partial x_{0}}{\partial v} \\
\underset{i}{\Sigma}\left(\frac{\partial x_{i}}{\partial v}\right)^{2}=G+\left(\frac{\partial x_{0}}{\partial v}\right)^{2}, & \underset{i}{\Sigma}\left(x_{i}+\xi_{i}\right)^{2}=\left(x_{0}+\xi_{0}\right)^{2} .
\end{array}
$$

(**) Il segno negativo della curvatura media dipende dal segno attribuito alle nelle (t l). 
Per completare queste osservazioni diciamo ancora che in geometria non euclidea possiamo eseguire la rappresentazione di Gauss in altri due modi corrispondenti al caso in cui il punto fisso $O$ dello spazio, anzichè reale e a distanza finita, sia reale all'infinito $o$ in fine ideale ed intercettando i sistemi di raggi paralleli alle normali della superficie $S$ con una superficie ortogonale ai raggi. Se il centro $O$ è all'infinito, le superficie normali ai raggi sono orisfere; quando invece $O$ è ideale fra le superficie normali ai raggi vi ha un piano (non euclideo) sul quale la $S$ viene rappresentata colla legge di parallelismo (non euclideo) delle normali nei punti corrispondenti. Le proprietà delle rappresentazioni ora accennate sono affatto analoghe alle superiori e possono anzi dedursi da questo adoperando la consueta rappresentazione conforme.

\section{$\S 12$.}

\section{Le nuove superficie a linee di curvatura isoterme.}

I risultati ultimamente conseguiti possono facilmente trasportarsi in geometria ordinaria. Serviamoci per ciò di quella rappresentazione conforme sul semi-spazio $z>0$, nella quale l'elemento lineare dello spazio iperbolico è dato da:

$$
d s^{2}=\frac{d x^{2}+d y^{2}+d z^{2}}{z^{2}}
$$

e consideriamo le superficie immagini di quelle a curvatura media $=2$. Ri.tenendo per la immagine, che pensiamo rappresentata dall'equazione:

$$
z=\approx(x, y)
$$

le ordinarie notazioni di Mones, la curvatura media della superficie obiettiva nello spazio iperbolico è dato da:

$$
\frac{1}{p_{1}}+\frac{1}{v_{0}}=\frac{z}{\left(1+p^{2}+q^{2}\right)^{\frac{3}{2}}}\left\{\left(1+q^{2}\right) v-2 p q s+\left(1+p^{2}\right) t+2 \frac{1+p^{2}+q^{2}}{z}\right\} \text {. }
$$

Ne segue che le superficie dello spazio ordinario domandato sono gli integrali delle equazioni a derivate parziali :

$$
\frac{\left(1+q^{2}\right) r-2 p q s+\left(1+p^{2}\right) t}{\left(1+p^{2}+q^{2}\right)^{\frac{3}{2}}}+\frac{2}{z}\left[\frac{1}{\sqrt{1+p^{2}+q^{2}}} \pm 1\right]=0 .
$$


I risultati ottenuti permettono d'integrare completamente questa equazione riducendola all'equazione di LiouviLle:

$$
\frac{\partial^{2} \theta}{\partial u^{2}}+\frac{\partial^{z} \theta}{\partial v^{2}}=e^{-2 \theta} \text {. }
$$

Tutte queste superficie (46, posseggono linee di curvatura isoterme e costituiscono una nuova classe di superficie isotermiche.

Possiamo in fine caratterizzare le superficie (46) mediante una proprietà geometrica che traduce, nello spazio ordinario, la proprietà di rappresentazione sferica di cui godono le obiettive $(\S 11)$ e ciò precisamente nel caso in cui il centro della sfera rappresentativa trovasi all'infinito. Ad una tale superficie $S$ integrale della $(46)$ conduciamo in ogni punto $M$ il circolo normale alla $S$ ed al piano $z=0$ e consideriamo i due punti $A, A^{\prime}$ in cui questo eircolo sega il piano. Otteniamo cosl sul piano $z=0$ due rappresentazioni di S, riguardando come punti corrispondenti $M, A$ ovvero $M, A^{\prime}$. Per le nostre superficie $S$ e per queste soltanto (oltre le sfere) una delle due rappresentazioni riesce conforme. 